\title{
ON THE MOTIVIC DONALDSON-THOMAS INVARIANTS OF QUIVERS WITH POTENTIALS
}

\author{
SERGey Mozgovoy
}

\begin{abstract}
We study motivic Donaldson-Thomas invariants for a class of quivers with potentials using the strategy of Behrend et al. [1]. This class includes quivers with potentials arising from consistent brane tilings and quivers with zero potential. Our construction is an alternative to the constructions of Kontsevich and Soibelman [8, 9]. We construct an integration map from the equivariant Hall algebra to the quantum torus and show that our motivic Donaldson-Thomas invariants are images of the natural elements in the equivariant Hall algebra. We show that the inegration map is an algebra homomorphism and use this fact to prove the Harder-Narasimhan relation for the motivic Donaldson-Thomas invariants.
\end{abstract}

\section{Introduction}

The goal of this paper is to study the motivic Donaldson-Thomas invariants for some class of quivers with (polynomial) potentials $(Q, W)$ using the approach of Behrend et al. [1]. These invariants are constructed using the motivic vanishing cycles of functions on smooth moduli spaces of stable quiver representations. The function $w: M_{\theta}^{\text {st }}(Q, \alpha) \rightarrow \mathbb{C}$ in question is the trace of the potential. It was proved in [1] that if $w$ is equivariant with respect to an appropriate torus action, then the motivic vanishing cycle of $w$ can be computed as

$$
\left[\varphi_{w}\right]=\left[w^{-1}(1)\right]-\left[w^{-1}(0)\right] .
$$

We will show that under certain conditions on the potential, we can introduce a weight function on the arrows, so that the corresponding torus action on the moduli space will satisfy all the required conditions. Therefore, the above equation will hold in this situation. Using the right-hand side of this equation for the definition of the motivic Donaldson-Thomas invariants and organizing these invariants as elements of the quantum torus, we will show that they can be obtained as images of some natural elements of the equivariant Hall algebra of the quiver $Q$ with respect to an algebra homomorphism (called an integration map) from the equivariant Hall algebra to the quantum torus. Our integration map is closely related to the integration map of Reineke [15] from the whole Hall algebra of $Q$ to the quantum torus. These maps coincide in the case of a trivial potential (see $[9,11]$ on the discussion of the motivic Donaldson-Thomas invariants in this case). In fact, the integration map of Reineke is an important ingredient in our construction.

We should stress, that our construction is quite different from the construction of Kontsevich and Soibelman [8], where an integration map from the Hall algebra of the category of modules over the Jacobian algebra to the quantum torus was defined. Our

Received by the editors June 28, 2011. 
approach is probably less natural, because we use the Hall algebra of the category of quiver representations in order to define some invariants of the moduli spaces of modules over the Jacobian algebra. However, it does the job - all the constructions are quite elementary, the algebra homomorphism property of the integration map is almost obvious, and the relations in the equivariant Hall algebra of the quiver (e.g. the Harder-Narasimhan relations) can be translated to the relations in the quantum torus, thus giving us relations between motivic Donaldson-Thomas invariants for different stability parameters. In the last part of the paper, we will see how our constructions can be generalized to arbitrary potentials, at least over finite fields. We expect that our invariants agree with the invariants of Kontsevich and Soibelman. For more discussion on this subject see [1, Section 2.5] and [4, Section 7.3].

While preparing this paper, I was informed by Kentaro Nagao on his related work [14] on the extension of the approach from [1] to more general quivers with potentials.

\section{Preliminaries}

2.1. Bilinear forms related to quivers. Let $Q=\left(Q_{0}, Q_{1}\right)$ be a quiver. We define the Euler-Ringel form to be the bilinear form on $\mathbb{Z}^{Q_{0}}$ given by

$$
\chi(\alpha, \beta)=\sum_{i \in Q_{0}} \alpha_{i} \beta_{i}-\sum_{a: i \rightarrow j} \alpha_{i} \beta_{j}, \quad \alpha, \beta \in \mathbb{Z}^{Q_{0}} .
$$

We define the skew-symmetric form

$$
\langle\alpha, \beta\rangle=\chi(\alpha, \beta)-\chi(\beta, \alpha) \quad \alpha, \beta \in \mathbb{Z}^{Q_{0}} .
$$

We define the Tits form $T(\alpha)=\chi(\alpha, \alpha), \alpha \in \mathbb{Z}^{Q_{0}}$.

2.2. Representations of quivers with potentials. Let $(Q, W)$ be a quiver with potential and let $J_{W}=k Q /(\partial W)$ be the corresponding Jacobian algebra over a field $k$. A representation $M$ of $Q$ over a field $k$ can be represented as

$$
M=\left(\left(M_{i}\right)_{i \in Q_{0}},\left(M_{a}\right)_{a \in Q_{1}}\right),
$$

where $M_{i}$ are $k$-vector spaces and $M_{a}: M_{s(a)} \rightarrow M_{t(a)}$ are linear maps (for any arrow $a \in Q_{1}$, we denote its source by $s(a)$ and denote its target by $\left.t(a)\right)$. Let $W=\sum a_{u} u$, where the sum runs over a finite number of cycles $u$ in $Q$. We define

$$
w(M)=\sum a_{u} \operatorname{tr}\left(M_{u}\right),
$$

where for any path $u=a_{1} \ldots a_{n}$, we define $M_{u}=M_{a_{1}} \ldots M_{a_{n}}$. Note that for any exact sequence of representations of $Q$

$$
0 \rightarrow N \rightarrow X \rightarrow M \rightarrow 0
$$

we have $w(X)=w(M)+w(N)$. Therefore, we get a map

$$
w: K_{0}(\operatorname{Rep}(Q, k)) \rightarrow k .
$$

For any $\alpha \in \mathbb{Z}^{Q_{0}}$, we define the space of representations

$$
R(Q, \alpha)=\bigoplus_{a: i \rightarrow j} \operatorname{Hom}\left(k^{\alpha_{i}}, k^{\alpha_{j}}\right),
$$


where the sum runs over all arrows of $Q$. There is a map

$$
w: R(Q, \alpha) \rightarrow k, \quad M \mapsto w(M),
$$

which is invariant under the action of $\mathrm{GL}_{\alpha}(k)=\prod_{i \in Q_{0}} \mathrm{GL}_{\alpha_{i}}(k)$ on $R(Q, \alpha)$ by conjugation. The following result is well-known [18, Proposition 3.8]

Lemma 2.1. A representation $M \in R(\alpha, \mathbb{C})$ is in the degeneracy locus of $w$ (i.e., $d w(M)=0)$ if and only if $M$ is a representation of the Jacobian algebra $J_{W}$.

2.3. Moduli spaces. Let $\theta \in \mathbb{R}^{Q_{0}}$ be some fixed vector. For any $\alpha \in \mathbb{N}^{Q_{0}} \backslash\{0\}$, we define

$$
\mu_{\theta}(\alpha)=\frac{\theta \cdot \alpha}{\sum \alpha_{i}}
$$

For any nonzero $Q$-representation $M$, we define $\mu_{\theta}(M)=\mu_{\theta}(\underline{\operatorname{dim}} M)$, where $\underline{\operatorname{dim}} M=$ $\left(\operatorname{dim} M_{i}\right)_{i \in Q_{0}} \in \mathbb{N}^{Q_{0}}$ is the dimension vector of $M$. We say that a representation $M$ is semistable (resp. stable) if for any $0 \neq N \subsetneq M$ we have $\mu_{\theta}(N) \leq \mu_{\theta}(M)$ (resp. $\left.\mu_{\theta}(N)<\mu_{\theta}(M)\right)$.

Let $M_{\theta}^{\text {sst }}(Q, \alpha)$ (resp. $\left.M_{\theta}^{\text {st }}(Q, \alpha)\right)$ be the moduli space of $\theta$-semistable (resp. stable) representations of $Q$ having dimension vector $\alpha$. Let $\mathcal{M}_{\theta}^{\text {sst }}(Q, \alpha)$ be the stack of $\theta$-semistable representations of $Q$ of dimension $\alpha$. The potential map $w: R(Q, \alpha) \rightarrow \mathbb{C}$ descends to $w: M_{\theta}^{\text {sst }}(Q, \alpha) \rightarrow k$ and $\tilde{w}: \mathcal{M}_{\theta}^{\text {sst }}(Q, \alpha) \rightarrow k$ (the reason is that $w$ is additive with respect to exact sequences of modules and therefore $w$ is constant on the classes of $S$-equivalence). It follows from Lemma 2.1 that the degeneracy locus of $w: M_{\theta}^{\text {st }}(Q, \alpha) \rightarrow k$ coincides with the moduli space $M_{\theta}^{\text {st }}\left(J_{W}, \alpha\right)$ of $\theta$-stable $J_{W}$-modules having dimension vector $\alpha$.

We say that $\theta$ is $\alpha$-generic if for any $0<\beta<\alpha$ we have $\mu_{\theta}(\beta) \neq \mu_{\theta}(\alpha)$. Then any semistable $Q$-representation of dimension $\alpha$ is automatically stable.

2.4. Weights. Let wt : $Q_{1} \rightarrow \mathbb{N}$ be some map, which we will call a weight function. For any path $u=a_{1} \cdots a_{n}$ we $\operatorname{define} \operatorname{wt}(u)=\sum \operatorname{wt} a_{i}$. We extend the weight function also to wt: $\mathbb{Z}^{Q_{1}} \rightarrow \mathbb{Z}$ by linearity.

Remark 2.2. Throughout this paper we assume that the potential $W$ is homogeneous of weight 1 with respect to wt. This means that for $W=\sum_{u} a_{u} u$ we have $\operatorname{wt}(u)=1$ whenever $a_{u} \neq 0$.

Remark 2.3. Such choice of weight function is always possible for quivers with potentials arising from consistent brane tilings [13]. For example, we can choose a weight function corresponding to the perfect matching of the associated bipartite graph (define the weight of an arrow to be equal 1 if it is in the perfect matching and zero otherwise).

For any representation $M$ and an element $t \in \mathbb{G}_{\mathrm{m}}$, we define a new representation $t M$ as follows:

$$
(t M)_{i}=M_{i}, \quad i \in Q_{0}, \quad(t M)_{a}=t^{\mathrm{wt}(a)} M_{a}, \quad a \in Q_{1} .
$$

This defines action of $\mathbb{G}_{\mathrm{m}}$ on $R(Q, \alpha)$ and on $M_{\theta}^{\text {sst }}(Q, \alpha)$. If $M$ is a representation of the Jacobian algebra $J_{W}$ then so also is $t M$. Note that

$$
w(t M)=t w(M)
$$




\section{Motivic vanishing cycle}

Let $K_{0}\left(\operatorname{Var}_{\mathbb{C}}\right)$ be the Grothendieck group of varieties $[1,5]$. It has a natural ring structure. Define the motivic ring to be $\mathcal{M}_{\mathbb{C}}=K_{0}\left(\operatorname{Var}_{\mathbb{C}}\right)\left[\mathbb{L}^{-\frac{1}{2}}\right]$. Similarly, let $\mathcal{M}_{\mathbb{C}}^{\hat{\mu}}$ be the monodromic motivic ring $[1,5]$. For any regular function $f: X \rightarrow \mathbb{C}$ on a smooth variety $X$, Denef and Loeser [5] defined the motivic vanishing cycle $\left[\varphi_{f}\right] \in \mathcal{M}_{\mathbb{C}}^{\hat{\mu}}$ of $f$. The following result can be used to compute $\left[\varphi_{f}\right]$.

Theorem 3.1 ([1, Proposition 1.11]). Let $f: X \rightarrow \mathbb{C}$ be a regular function on $a$ smooth variety $X$. Assume that $X$ admits a $\mathbb{C}^{*}$-action such that $f$ is $\mathbb{C}^{*}$-equivariant i.e. , $f(t x)=t f(x)$ for $t \in \mathbb{C}^{*}, x \in X$, and such that there exist limits $\lim _{t \rightarrow 0} t x$ for all $x \in X$. Then

$$
\left[\varphi_{f}\right]=\left[f^{-1}(1)\right]-\left[f^{-1}(0)\right] \in \mathcal{M}_{\mathbb{C}} \subset \mathcal{M}_{\mathbb{C}}^{\hat{\mu}}
$$

Remark 3.2. In $\left[1\right.$, Proposition 1.11] there is also a requirement that $X^{\mathbb{C}^{*}}$ is compact. But, as it is mentioned after [1, Proposition 1.11], this condition can be dropped.

Let $(Q, W)$ be a quiver with a potential and let wt $: Q_{1} \rightarrow \mathbb{N}$ be a weight function as in Remark 2.2. Given $\alpha \in \mathbb{N}^{Q_{0}}$ and stability parameter $\theta \in \mathbb{R}^{Q_{0}}$, the moduli space $M_{\theta}^{\text {sst }}(Q, \alpha)$ has a $\mathbb{C}^{*}$-action induced by the weight function. We will show in Lemmma 3.4 that this action satisfies the conditions of Theorem 3.1 for the function $w: M_{\theta}^{\text {sst }}(Q, \alpha) \rightarrow \mathbb{C}$ if $\theta$ is $\alpha$-generic.

Remark 3.3. It follows from the assumption that $\theta$ is $\alpha$-generic that all modules in $M_{\theta}^{\text {sst }}(Q, \alpha)$ are $\theta$-stable and that $M_{\theta}^{\text {sst }}(Q, \alpha)$ is smooth. The fact that

$$
w: M_{\theta}^{\mathrm{sst}}(Q, \alpha) \rightarrow \mathbb{C}
$$

is $\mathbb{C}^{*}$-equivariant follows from $(2.1)$.

Lemma 3.4. Let $\alpha \in \mathbb{N}^{Q_{0}}$ and let $\theta \in \mathbb{R}^{Q_{0}}$ be $\alpha$-generic. Then for any $[M] \in$ $M_{\theta}^{\text {sst }}(Q, \alpha)$ there exists the limit $\lim _{t \rightarrow 0} t[M]$.

Proof. Let $M_{0}(Q, \alpha)=R(Q, \alpha) / / \mathrm{GL}_{\alpha}$. Then $M_{0}(Q, \alpha)$ is affine and there is a canonical projective map $\pi: M_{\theta}^{\text {sst }}(Q, \alpha) \rightarrow M_{0}(Q, \alpha)$ (see [7]). For any $[M] \in M_{0}(Q, \alpha)$ there exists the $\operatorname{limit}_{t \rightarrow 0} t[M]$ as it exists already in $R(Q, \alpha)$. Indeed, for any point $M \in R(Q, \alpha)$ we have $\lim _{t \rightarrow 0} t M=M^{\prime}$, where $M_{a}^{\prime}=0$ if $\operatorname{wt}(a)>0$ and $M_{a}^{\prime}=M_{a}$ if $\operatorname{wt}(a)=0$ for $a \in Q_{1}$. Now our statement follows from the properness of $\pi$.

Remark 3.5. It is not true in general, that for any $M \in R_{\theta}^{\text {sst }}(Q, \alpha) \subset R(Q, \alpha)$ there exists the $\operatorname{limit}_{\lim _{t \rightarrow 0} t M}$ in $R_{\theta}^{\text {sst }}(Q, \alpha)$. Therefore we do not formulate analogous statement for the moduli stacks of representations. For example, consider the quiver $Q$ with two vertices 1,2 and one arrow $a: 1 \rightarrow 2$. Let $\alpha=(1,1), \theta=(1,-1)$ and let the action of $\mathbb{C}^{*}$ on $R(Q, \alpha)$ be given by multiplication. Then $M_{\theta}^{\text {sst }}(Q, \alpha)$ consists of one point $[M]$, which is an isomorphism class of the representation $M=[\mathbb{C} \stackrel{1}{\rightarrow} \mathbb{C}]$. The $\operatorname{limit}_{\lim _{t \rightarrow 0}} t[M]$ exists and coincides with $[M]$. On the other hand, $R_{\theta}^{\text {sst }}(Q, \alpha)$ consists of representations $M_{s}=[\mathbb{C} \stackrel{s}{\rightarrow} \mathbb{C}], s \in \mathbb{C}^{*}$, and the limit $\lim _{t \rightarrow 0} t M_{1}=[\mathbb{C} \stackrel{0}{\rightarrow} \mathbb{C}]$ is not contained in $R_{\theta}^{\mathrm{sst}}(Q, \alpha)$.

In the next theorem, we will show that $M_{\theta}^{\text {sst }}(Q, \alpha)^{\mathbb{C}^{*}}$ is compact. It is not necessary for the other results of this paper. The additional condition that wt : $Q_{1} \rightarrow \mathbb{N}$ is 
positive on every cycle is satisfied, in particular, for quivers with potentials arising from brane tilings; see Remark 2.3.

Theorem 3.6. Let $\alpha \in \mathbb{N}^{Q_{0}}$ and let $\theta \in \mathbb{R}^{Q_{0}}$ be $\alpha$-generic. Assume that the weight function wt $: Q_{1} \rightarrow \mathbb{N}$ is positive on every cycle. Then the subvariety of $\mathbb{C}^{*}$-invariant points of $M_{\theta}^{\mathrm{sst}}(Q, \alpha)$ is a projective variety.

Proof. We follow the strategy of [17]. In the first part of the proof we will consider a general weight function wt $: Q_{1} \rightarrow \Lambda$, where $\Lambda$ is a free finitely generated abelian group. It induces an action of the torus $T=\operatorname{Hom}_{\mathbb{Z}}\left(\Lambda, \mathbb{C}^{*}\right)$ on $\mathcal{M}_{\theta}^{\text {sst }}(Q, \alpha)$. The group $\Lambda$ is a group of characters of $T$ and we will denote the pairing between $T$ and $\Lambda$ by $(t, \lambda) \mapsto t^{\lambda}$.

Let $[M] \in M_{\theta}^{\text {sst }}(Q, \alpha)$ be some $T$-invariant point. It is represented by some $\theta$-stable $M \in R(Q, \alpha)$. There is a natural action of the group $G_{\alpha}=\prod_{i \in Q_{0}} \mathrm{GL}_{\alpha_{i}}$ on $R(Q, \alpha)$ by conjugation. As $M$ is stable, the stabilizer of $M$ is given by $\mathbb{C}^{*} \subset G_{\alpha}$ embedded diagonally. Let $P G_{\alpha}=G_{\alpha} / \mathbb{C}^{*}$. By our assumption for any $t \in T$ there exists some $g=\left(g_{i}\right)_{i \in Q_{0}} \in \mathrm{GL}_{\alpha}$ such that for any arrow $a: i \rightarrow j$ in $Q$

$$
(t M)_{a}=g_{j} M_{a} g_{i}^{-1} .
$$

Let $H \subset T \times P G_{\alpha}$ be the subgroup of all elements $(t, g)$ satisfying this condition. Then $p_{1}: H \rightarrow T$ is surjective by our assumption. On the other hand, its kernel is trivial and therefore $p_{1}$ is an isomorphism. The composition

$$
\bar{\psi}: p_{2} \circ p_{1}^{-1}: T \rightarrow P G_{\alpha}
$$

can be lifted to $\psi: T \rightarrow G_{\alpha}[19]$. We split $\psi$ to components $\psi_{i}: T \rightarrow \operatorname{GL}\left(M_{i}\right), i \in Q_{0}$ and decompose every $M_{i}$ with respect to the character group $\Lambda$ of $T$

$$
M_{i}=\bigoplus_{\lambda \in \Lambda} M_{i, \lambda}, \quad i \in Q_{0}
$$

One can see that for any arrow $a: i \rightarrow j$ we have

$$
M_{a}\left(M_{i, \lambda}\right) \subset M_{j, \lambda+\operatorname{wt} a} .
$$

Conversely, assume that such a grading on $M$ exists. The $\Lambda$-grading of $M_{i}$ induces a group homomorphism $\psi_{i}: T \rightarrow \mathrm{GL}\left(M_{i}\right)$ given by

$$
\psi_{i}(t) m=t^{\lambda} m, \quad m \in M_{i, \lambda} .
$$

Given $t \in T$, let $g=\left(g_{i}\right)_{i \in Q_{0}}=\left(\psi_{i}(t)\right)_{i \in Q_{0}}$. Equation (3.1) implies that for any arrow $a: i \rightarrow j$ and any $m \in M_{i, \lambda}$ we have

$$
(t M)_{a} m=t^{\mathrm{wt} a} M_{a} m=\psi_{j}(t) M_{a} \psi_{i}(t)^{-1} m=g_{j} M_{a} g_{i}^{-1} m .
$$

This means that $M$ is $T$-invariant in $M_{\theta}^{\mathrm{sst}}(Q, \alpha)$.

If $\psi^{\prime}$ is a different lift of $\bar{\psi}$, then $\psi^{\prime} \psi^{-1}: T \rightarrow \mathbb{C}^{*}$. It can be considered as an element in $\Lambda$. This element defines a shift in $\Lambda$-gradings of $M$ corresponding to $\psi$ and $\psi^{\prime}$. Thus, a $\Lambda$-grading of a $T$-invariant representation in $M_{\theta}^{\text {sst }}(Q, \alpha)$ is defined only up 
to shifts by elements in $\Lambda$. Using this shift we can assume that $M_{i_{0}, 0} \neq 0$, for some $i_{0} \in Q_{0}$.

Let now $\Lambda=\mathbb{Z}$ and wt $: Q_{1} \rightarrow \mathbb{Z}$ be as in the assumption of the theorem. There exists a boundary $N$ such that $M_{i, n}=0$ for $i \in Q_{0},|n|>N$. For example, we can take

$$
N=|\alpha| m, \quad|\alpha|=\sum_{i \in Q_{0}} \alpha_{i}=\operatorname{dim} M, \quad m=\max _{a \in Q_{1}} \operatorname{wt} a .
$$

Indeed, if there exists say $n_{0}>N$ such that $M_{i, n_{0}} \neq 0$ for some $i \in Q_{0}$, then there exists $0 \leq k<k+m<n_{0}$ such that $M_{i, n}=0$ for all $i \in Q_{0}, k+1 \leq n \leq k+m$. However, then $M$ is a direct sum of two submodules

$$
\bigoplus_{i \in Q_{0}, n \leq k} M_{i, n}, \quad \bigoplus_{i \in Q_{0}, n>k+m} M_{i, n}
$$

where the first submodule is nonzero because $M_{i_{0}, 0} \neq 0$. This contradicts the stability of $M$.

The grading of $M$ allows us to construct a representation $\widehat{M}$ of the following quiver $\widehat{Q}$. Its vertices are pairs

$$
(i, n), \quad i \in Q_{0}, \quad-N \leq n \leq N
$$

and its arrows are pairs

$$
(a, n):(i, n) \rightarrow(j, n+\operatorname{wt}(a)), \quad(a: i \rightarrow j) \in Q_{1}, \quad-N \leq n \leq n+\operatorname{wt} a \leq N .
$$

The dimension vector of $\widehat{M}$ equals $\widehat{\alpha} \in \mathbb{Z}^{\widehat{Q}_{1}}$ given by $\widehat{\alpha}_{i, n}=\operatorname{dim} M_{i, n}$.

It is clear that $\widehat{M}$ is stable with respect to the stability condition $\widehat{\theta}$ defined by $\widehat{\theta}_{i, n}=\theta_{i}$. Conversely, given a $\widehat{\theta}$-stable representation $\widehat{M}$ of the quiver $\widehat{Q}$ of dimension $\widehat{\alpha}$, we can construct a representation $M$ of the quiver $Q$ of dimension $\alpha$ which is fixed by $\mathbb{C}^{*}$. We claim, that $M$ is $\theta$-stable (or, equivalently, $\theta$-semistable). If this is wrong then there exists a destabilizing semistable submodule $N \subset M$ coming from the Harder-Narasimhan filtration. It follows from the uniqueness of the HarderNarasimhan filtration that $N$ is actually $\mathbb{C}^{*}$-invariant. Let $U \subset N$ be some stable submodule. For any $t \in \mathbb{C}^{*}$, the stable representation $t U$ is a submodule of $t N \simeq N$. It follows that $t U, t \in \mathbb{C}^{*}$, form a direct sum in $N$ (because they are simple objects in the category of semistable modules having the same slope as $N)$. As $N$ is finitedimensional, the orbit $t U, t \in \mathbb{C}^{*}$, in the moduli space of stable modules should be finite. However, it is an image of a connected group $\mathbb{C}^{*}$, so the orbit consists of just one element. This implies that $U$ is $\mathbb{C}^{*}$-invariant. The same argument as earlier for the module $M$ shows that $U$ has a $\Lambda$-grading. We can choose this grading to be compatible with the grading of $M$. Then $U$ can be considered as a representation $\widehat{U}$ of the quiver $\widehat{Q}$. Therefore $\widehat{U}$ is a destabilizing submodule of $\widehat{M}$ and this contradicts to the stability of $\widehat{M}$.

We have shown that the subvariety of $\mathbb{C}^{*}$-invariant points of $M_{\theta}^{\text {sst }}(Q, \alpha)$ can be identified with the moduli space $M_{\widehat{\theta}}^{\text {sst }}(\widehat{Q}, \widehat{\alpha})$ (note that $\widehat{\theta}$ is $\widehat{\alpha}$-generic). Note that the quiver $\widehat{Q}$ is acyclic, because any cycle in $\widehat{Q}$ would project to a cycle in $Q$ and all cycles in $Q$ have positive weights by our assumptions. This implies that $M_{\widehat{\theta}}^{\text {sst }}(\widehat{Q}, \widehat{\alpha})$ is projective and the theorem is proved. 


\section{Motivic Donaldson-Thomas invariants}

Let $(Q, W)$ be a quiver with a potential and let wt $: Q_{1} \rightarrow \mathbb{N}$ be a weight function satisfying the condition from Remark 2.2. Let $\alpha \in \mathbb{N}^{Q_{0}}$ and let $\theta \in \mathbb{R}^{Q_{0}}$ be $\alpha$-generic. Consider the trace of the potential (also for $\theta$ non- $\alpha$-generic) for the moduli space and the moduli stack

$$
w: M_{\theta}^{\text {sst }}(Q, \alpha) \rightarrow \mathbb{C}, \quad \tilde{w}: \mathcal{M}_{\theta}^{\text {sst }}(Q, \alpha) \rightarrow \mathbb{C} .
$$

We can write the moduli stack $\mathcal{M}_{\theta}^{\text {sst }}(Q, \alpha)$ as the global quotient stack $\left[M_{\theta}^{\text {sst }}(Q, \alpha) / \mathbb{C}^{*}\right]$ with a trivial action of $\mathbb{C}^{*}$ (indeed, the group $G_{\alpha}=\mathrm{GL}_{\alpha} / \mathbb{C}^{*}$ acts freely on $R_{\theta}^{\text {sst }}(Q, \alpha)$ and therefore the quotient stack $\left[R_{\theta}^{\text {sst }}(Q, \alpha) / G_{\alpha}\right]$ is isomorphic to $M_{\theta}^{\text {sst }}(Q, \alpha)$ and the quotient stack $\left[R_{\theta}^{\text {sst }}(Q, \alpha) / \mathrm{GL}_{\alpha}\right]$ is isomorphic to $\left[M_{\theta}^{\text {sst }}(Q, \alpha) /\right.$ $\left.\left.\mathbb{C}^{*}\right]\right)$. We refer to [2] for the definition of the motive of an algebraic stack. In particular, we have

$$
\left[\mathcal{M}_{\theta}^{\text {sst }}(Q, \alpha)\right]=\frac{\left[M_{\theta}^{\text {sst }}(Q, \alpha)\right]}{\left[\mathbb{C}^{*}\right]}=\frac{\left[M_{\theta}^{\text {sst }}(Q, \alpha)\right]}{\mathbb{L}-1} .
$$

To avoid the general definition of the motivic vanishing cycle for stacks, we define

$$
\left[\varphi_{\tilde{w}}\right]=\frac{\left[\varphi_{w}\right]}{\mathbb{L}-1},
$$

see Section 3. Following [1, Definition 1.13], we formulate

Definition 4.1. Let $\alpha \in \mathbb{N}^{Q_{0}}$ and let $\theta \in \mathbb{R}^{Q_{0}}$ be $\alpha$-generic. We define the virtual motive $A_{\alpha}^{\theta}$ of the moduli stack $\mathcal{M}_{\theta}^{\text {sst }}\left(J_{W}, \alpha\right)$ by the formula

$$
A_{\alpha}^{\theta}=\left[\mathcal{M}_{\theta}^{\mathrm{sst}}\left(J_{W}, \alpha\right)\right]_{v i r}:=-\left(-\mathbb{L}^{\frac{1}{2}}\right)^{-\operatorname{dim} \mathcal{M}_{\theta}^{\mathrm{sst}}(Q, \alpha)}\left[\varphi_{\tilde{w}}\right] .
$$

Remark 4.2. Note that

$$
\operatorname{dim} \mathcal{M}_{\theta}^{\mathrm{sst}}(Q, \alpha)=\operatorname{dim} M_{\theta}^{\mathrm{sst}}(Q, \alpha)-1=-T(\alpha),
$$

where $T(\alpha)=\chi(\alpha, \alpha)$ is the Tits form of the quiver $Q$.

Proposition 4.3. We have

$$
\left[\varphi_{w}\right]=\left[w^{-1}(1)\right]-\left[w^{-1}(0)\right] \in \mathcal{M}_{\mathbb{C}} .
$$

Proof. By our assumptions on the weight function, Remark 3.3, and Lemma 3.4, we can apply Theorem 3.1.

Corollary 4.4. We have

$$
A_{\alpha}^{\theta}=\left(-\mathbb{L}^{\frac{1}{2}}\right)^{T(\alpha)} \frac{\left[w^{-1}(0)\right]-\left[w^{-1}(1)\right]}{\mathbb{L}-1}=\left(-\mathbb{L}^{\frac{1}{2}}\right)^{T(\alpha)}\left(\left[\tilde{w}^{-1}(0)\right]-\left[\tilde{w}^{-1}(1)\right]\right) .
$$

Definition 4.5. For a not necessarily $\alpha$-generic stability parameter $\theta$, we define the virtual motive $A_{\alpha}^{\theta}$ of the moduli stack $\mathcal{M}_{\theta}^{\text {sst }}\left(J_{W}, \alpha\right)$ to be

$$
A_{\alpha}^{\theta}=\left[\mathcal{M}_{\theta}^{\text {sst }}\left(J_{W}, \alpha\right)\right]_{\mathrm{vir}}:=\left(-\mathbb{L}^{\frac{1}{2}}\right)^{T(\alpha)}\left(\left[\tilde{w}^{-1}(0)\right]-\left[\tilde{w}^{-1}(1)\right]\right) .
$$

For any $\mu \in \mathbb{R}$, we define the motivic Donaldson-Thomas series (they are elements of the motivic quantum torus, see the next section)

$$
A_{\mu}^{\theta}=\sum_{\mu_{\theta}(\alpha)=\mu} A_{\alpha}^{\theta} x^{\alpha} .
$$


Note that Equation (4.2) can be interpreted also over finite fields, where instead of the motive $\left[\tilde{w}^{-1}(t)\right]$ we write the number of points in the corresponding fiber. In the next section, we will use this observation in order to work with Hall algebras over finite fields (which are technically less involved than the motivic Hall algebras). This is done to make the exposition clearer. With some additional effort our results can be translated back to motivic Hall algebras and motivic Donaldson-Thomas invariants [12].

\section{Equivariant Hall algebra}

Let $H$ be the Hall algebra of the category of representations of the quiver $Q$ over a finite field $k=\mathbb{F}_{q}$ (we use the conventions of [8] for the multiplication and this gives an algebra opposite to the usual Ringel-Hall algebra). The basis of $H$ as a vector space consists of all isomorphism classes of representations of $Q$ over $\mathbb{F}_{q}$. Multiplication is given by the rule

$$
[N] \circ[M]=\sum_{[X]} F_{M N}^{X}[X]
$$

where

$$
F_{M N}^{X}=\#\{U \subset X \mid U \simeq N, X / U \simeq M\} .
$$

Let $H_{\text {eq }} \subset H$ be a subalgebra consisting of elements $f=\sum a_{M}[M]$ such that $a_{t M}=$ $a_{M}$ for any $t \in k^{*}$. We call $H_{\text {eq }}$ the equivariant Hall algebra. For any $f=\sum a_{M}[M] \in$ $H_{\text {eq }}$ and $t \in k$, we define $f_{t}=\sum_{w(M)=t} a_{M}[M]$. The algebras $H, H_{\text {eq }}$ are graded by the dimension of representations. We denote by $\widehat{H}, \widehat{H}_{\text {eq }}$ the corresponding completions.

Let $\mathbb{T}$ be the quantum torus of the quiver $Q$ (more precisely this will be just some part of the quantum torus). As a vector space it is

$$
\mathbb{Q}\left(q^{\frac{1}{2}}\right) \llbracket x_{1}, \ldots, x_{r} \rrbracket,
$$

where $r=\# Q_{0}$. Multiplication is given by

$$
x^{\alpha} \circ x^{\beta}=\left(-q^{\frac{1}{2}}\right)^{\langle\alpha, \beta\rangle} x^{\alpha+\beta},
$$

where $\langle-,-\rangle$ is the skew-symmetric form of the quiver $Q$, see Section 2.1. It was shown by Markus Reineke [16] that there exists an algebra homomorphism

$$
I: \widehat{H} \rightarrow \mathbb{T}, \quad[M] \mapsto \frac{\left(-q^{\frac{1}{2}}\right)^{T(\underline{\operatorname{dim}} M)}}{\# \operatorname{Aut} M} x \underline{\operatorname{dim} M} .
$$

Remark 5.1. Similarly, one can define the motivic Hall algebra of representations over $Q$ (see e.g. $[6,8,3]$ ) and the quantum torus over the Grothendieck ring of the category of Chow motives [8], where multiplication is given by

$$
x^{\alpha} \circ x^{\beta}=\left(-\mathbb{L}^{\frac{1}{2}}\right)^{\langle\alpha, \beta\rangle} x^{\alpha+\beta} .
$$

There is an algebra homomorphism from the motivic Hall algebra to the motivic quantum torus similar to the above map.

Let $\tilde{w}: \mathcal{M}_{\theta}^{\text {sst }}(Q, \alpha) \rightarrow k$ be the trace of the potential. The invariants $A_{\alpha}^{\theta}$ defined in Equation (4.2) can be also defined over a finite field $\mathbb{F}_{q}$. The point count of the stack 
$\mathcal{M}_{\theta}^{\text {sst }}(Q, \alpha)$ (multiplied by $\left.\left(-q^{\frac{1}{2}}\right)^{T(\alpha)}\right)$ corresponds to $I\left(\tilde{A}_{\alpha}^{\theta}\right)$, where

$$
\tilde{A}_{\alpha}^{\theta}=\sum_{\substack{[M] \text { is } \theta-s s t \\ \underline{\operatorname{dim} M=\alpha}}}[M] \in \widehat{H}_{\mathrm{eq}} .
$$

The point count of $\tilde{w}^{-1}(t), t \in k,\left(\right.$ multiplied by $\left.\left(-q^{\frac{1}{2}}\right)^{T(\alpha)}\right)$ corresponds to $I\left(\left(\tilde{A}_{\alpha}^{\theta}\right)_{t}\right)$ (recall that for $f=\sum a_{M}[M]$, we define $\left.f_{t}=\sum_{w(M)=t} a_{M}[M]\right)$. Therefore, over a finite field $k=\mathbb{F}_{q}$, we define

$$
A_{\alpha}^{\theta} x^{\alpha}=I\left(\left(\tilde{A}_{\alpha}^{\theta}\right)_{0}\right)-I\left(\left(\tilde{A}_{\alpha}^{\theta}\right)_{1}\right)=I_{\mathrm{eq}}\left(\tilde{A}_{\alpha}^{\theta}\right),
$$

where the map $I_{\mathrm{eq}}: \widehat{H}_{\mathrm{eq}} \rightarrow \mathbb{T}$ is given by

$$
I_{\text {eq }}(f)=I\left(f_{0}\right)-I\left(f_{1}\right) .
$$

Proposition 5.2. The map $I_{\mathrm{eq}}: \widehat{H}_{\mathrm{eq}} \rightarrow \mathbb{T}$ is an algebra homomorphism.

Proof. For any $f \in H_{\text {eq }}, t \in k^{*}$ we have

$$
I\left(f_{t}\right)=\frac{I(f)-I\left(f_{0}\right)}{q-1}
$$

and therefore

$$
I_{\mathrm{eq}}(f)=\frac{q I\left(f_{0}\right)-I(f)}{q-1} .
$$

The map $w$ is additive with respect to exact sequences. Therefore

$$
(f g)_{0}=\sum_{t \in k} f_{t} g_{-t} .
$$

Let $F=I(f), F_{0}=I\left(f_{0}\right), G=I(g), G_{0}=I\left(g_{0}\right)$. Then

$$
I\left((f g)_{0}\right)=\sum_{t \in k} I\left(f_{t}\right) I\left(g_{-t}\right)=\frac{\left(F-F_{0}\right)\left(G-G_{0}\right)}{q-1}+F_{0} G_{0} .
$$

Therefore

$$
\begin{aligned}
I_{\mathrm{eq}}(f g) & =\frac{q I\left((f g)_{0}\right)-I(f g)}{q-1} \\
& =\frac{q(q-1) F_{0} G_{0}+q\left(F-F_{0}\right)\left(G-G_{0}\right)-(q-1) F G}{(q-1)^{2}} \\
& =\frac{q^{2} F_{0} G_{0}-q\left(F G_{0}+F_{0} G\right)+F G}{(q-1)^{2}} \\
& =\frac{\left(q F_{0}-F\right)\left(q G_{0}-G\right)}{(q-1)^{2}}=I_{\mathrm{eq}}(f) I_{\mathrm{eq}}(g) .
\end{aligned}
$$

Remark 5.3. Note that if $f=f_{0}$, then $I_{\text {eq }}(f)=I(f)$. This implies that the unit element is sent to the unit element by $I_{\text {eq }}$. If the potential $W$ is trivial then $I_{\text {eq }}=I$.

Definition 5.4. For any $\mu \in \mathbb{R}$, we define

$$
\tilde{A}_{\mu}^{\theta}=\sum_{\mu_{\theta}(\alpha)=\mu} \tilde{A}_{\alpha}^{\theta}=\sum_{\substack{[M] \text { is } \theta-s s t \\ \mu_{\theta}(\underline{\operatorname{dim}} M)=\mu}}[M] \in \widehat{H}_{\mathrm{eq}} .
$$


We define the motivic Donaldson-Thomas series

$$
A_{\mu}^{\theta}=I_{\mathrm{eq}}\left(\tilde{A}_{\mu}^{\theta}\right)=\sum_{\mu_{\theta}(\alpha)=\mu} A_{\alpha}^{\theta} x^{\alpha} .
$$

For $\theta=0$ and $\mu=0$, we denote $\tilde{A}_{\mu}^{\theta}$ (resp. $A_{\mu}^{\theta}$ and $A_{\alpha}^{\theta}$ ) just by $\tilde{A}$ (resp. $A$ and $A_{\alpha}$ ).

It follows from the Harder-Narasimhan filtration for $Q$-representations that, for any $\theta \in \mathbb{R}^{Q_{0}}$, we have

$$
\tilde{A}=\prod_{\mu}^{\leftarrow} \tilde{A}_{\mu}^{\theta} \in \widehat{H}_{\mathrm{eq}}
$$

where the product is taken in the decreasing order of $\mu \in \mathbb{R}$. Applying the algebra homomorphism $I_{\text {eq }}$, we obtain an analogous statement in the quantum torus.

Theorem 5.5 (Harder-Narasimhan relation). For any $\theta \in \mathbb{R}^{Q_{0}}$, we have

$$
A=\prod_{\mu}^{\leftarrow} A_{\mu}^{\theta}
$$

where the product is taken in the decreasing order of $\mu \in \mathbb{R}$.

This recursion formula can be solved using the approach of Markus Reineke [15] (see also [10, Theorem 3.2]).

Theorem 5.6. For any $\theta \in \mathbb{R}^{Q_{0}}$, we have

$$
A_{\alpha}^{\theta}=\sum_{\left(\alpha_{1}, \ldots, \alpha_{k}\right)}(-1)^{k-1}\left(-q^{\frac{1}{2}}\right)^{\sum_{i<j}\left\langle\alpha_{i}, \alpha_{j}\right\rangle} \prod_{i=1}^{k} A_{\alpha_{i}} .
$$

where the sum runs over all tuples $\left(\alpha_{1}, \ldots, \alpha_{k}\right)$ of vectors in $\mathbb{N}^{Q_{0}} \backslash\{0\}$ such that $\sum_{i=1}^{k} \alpha_{i}=\alpha$ and $\mu\left(\sum_{i=1}^{j} \alpha_{i}\right)>\mu(\alpha)$ for any $1 \leq j<k$.

Proof. According to the previous theorem, for any $\alpha \in \mathbb{N}^{Q_{0}} \backslash\{0\}$, we can write

$$
A_{\alpha} x^{\alpha}=\sum_{\left(\alpha_{1}, \ldots, \alpha_{k}\right)}\left(A_{\alpha_{1}}^{\theta} x^{\alpha_{1}}\right) \circ \cdots \circ\left(A_{\alpha_{k}}^{\theta} x^{\alpha_{k}}\right),
$$

where the sum runs over all tuples $\left(\alpha_{1}, \ldots, \alpha_{k}\right)$ of vectors in $\mathbb{N}^{Q_{0}} \backslash\{0\}$ such that $\sum_{i=1}^{k} \alpha_{i}=\alpha$ and $\mu\left(\alpha_{1}\right)>\cdots>\mu\left(\alpha_{k}\right)$. Applying [10, Theorem 3.2] we deduce that

$$
A_{\alpha}^{\theta} x^{\alpha}=\sum_{\left(\alpha_{1}, \ldots, \alpha_{k}\right)}(-1)^{k-1}\left(A_{\alpha_{1}} x^{\alpha_{1}}\right) \circ \cdots \circ\left(A_{\alpha_{k}} x^{\alpha_{k}}\right)
$$

where the sum runs over all tuples $\left(\alpha_{1}, \ldots, \alpha_{k}\right)$ of vectors in $\mathbb{N}^{Q_{0}} \backslash\{0\}$ such that $\sum_{i=1}^{k} \alpha_{i}=\alpha$ and $\mu\left(\sum_{i=1}^{j} \alpha_{i}\right)>\mu(\alpha)$ for any $1 \leq j<k$. The statement of the theorem follows now from the definition of the multiplication in the quantum torus $\mathbb{T}$.

Remark 5.7. It follows from the above results that if $A_{\alpha}$ are rational functions in $q^{\frac{1}{2}}$, for $\alpha \in \mathbb{N}^{Q_{0}}$, then so are also $A_{\alpha}^{\theta}$ for any stability parameter $\theta \in \mathbb{R}^{Q_{0}}$. 


\section{Quivers with arbitrary potentials}

As we explained earlier, the results of the previous section can be proved also in the motivic setting. In this section, however, we will work only with quiver representations over finite fields. Let $\mathbb{F}_{q}$ be some finite field. We extend scalars in the quantum torus and define

$$
\mathbb{T}=\mathbb{C} \llbracket x_{1}, \ldots, x_{r} \rrbracket
$$

with multiplication $x^{\alpha} \circ x^{\beta}=\left(-q^{\frac{1}{2}}\right)^{\langle\alpha, \beta\rangle} x^{\alpha+\beta}$.

Let $\psi: \mathbb{F}_{q} \rightarrow \mathbb{C}^{*}$ be some non-trivial character. Define the map

$$
I_{\psi}: \widehat{H} \rightarrow \mathbb{T}, \quad[M] \mapsto \psi(w(M)) I(M),
$$

where the map $I: \widehat{H} \rightarrow \mathbb{T}$ was defined in (5.1).

Lemma 6.1. The map $I_{\psi}: \widehat{H} \rightarrow \mathbb{T}$ is an algebra homomorphism.

Proof. The map $w$ is additive with respect to exact sequences. Therefore,

$$
\begin{aligned}
I_{\psi}([N] \circ[M]) & =I_{\psi}\left(\sum F_{M N}^{X} X\right) \\
& =\psi w(M) \psi w(N) I\left(\sum F_{M N}^{X}[X]\right) \\
& =\psi w(M) \psi w(N) I(N) I(M)=I_{\psi}(N) I_{\psi}(M) .
\end{aligned}
$$

Recall that in the previous section we have used the weight function wt: $Q_{1} \rightarrow \mathbb{Z}$ in order to define the action of $\mathbb{G}_{\mathrm{m}}$ on the quiver representations and to define the equivariant Hall algebra $H_{\text {eq }} \subset H$.

Lemma 6.2. For any $f \in \widehat{H}_{\mathrm{eq}}$, we have $I_{\mathrm{eq}}(f)=I_{\psi}(f)$.

Proof. It is enough to prove the statement for $f=\sum_{t \in \mathbb{F}_{q}^{*}}[t M]$, where $w(M) \neq 0$, and for $f=[M]$, where $w(M)=0$. In the first case, we have

$$
I_{\psi}(f)=\sum_{t \in \mathbb{F}_{q}^{*}} \psi w(t M) I(M)=\sum_{t \in \mathbb{F}_{q}^{*}} \psi(t) I(M)=-I(M)=\frac{I(f)}{1-q}=I_{\mathrm{eq}}(f) .
$$

In the second case, we have

$$
I_{\psi}(f)=I(M)=I_{\text {eq }}(f) .
$$

This lemma means that instead of using the homomorphism $I_{\mathrm{eq}}: \widehat{H}_{\mathrm{eq}} \rightarrow \mathbb{T}$ for the definition of Donaldson-Thomas series, we can use the homomorphism $I_{\psi}: \widehat{H} \rightarrow \mathbb{T}$. While the homomorphism $I_{\text {eq }}$ depends on the weight function wt $: Q_{1} \rightarrow \mathbb{Z}$ (more precisely, its domain $\widehat{H}_{\text {eq }}$ depends on wt), the homomorphism $I_{\psi}$ depends only on the character $\psi$ and its domain is the whole Hall algebra $\widehat{H}$. We can use $I_{\psi}$ to define the Donaldson-Thomas series for arbitrary potentials, as this approach does not require a weight function wt : $Q_{1} \rightarrow \mathbb{Z}$, which is compatible with the potential.

\section{Acknowledgments}

I would like to thank Tamas Hausel and Markus Reineke for many useful discussions. The authors research was supported by the EPSRC under grant EP/G027110/1. 


\section{References}

[1] K. Behrend, J. Bryan and B. Szendrői, Motivic degree zero Donaldson-Thomas invariants, Invent. Math. 192 (2013), 111-160.

[2] K. Behrend and A. Dhillon, On the motivic class of the stack of bundles, Adv. Math. 212(2) (2007), 617-644.

[3] T. Bridgeland, An introduction to motivic Hall algebras, Adv. Math. 229(1), (2012), 102-138.

[4] B. Davison, Invariance of orientation data for ind-constructible Calabi-Yau $A_{\infty}$ categories under derived equivalence, $\mathrm{PhD}$ thesis, University of Oxford, 2011.

[5] J. Denef and F. Loeser, Geometry on arc spaces of algebraic varieties, in 'European Congress of Mathematics', Vol. I (Barcelona, 2000), Progr. Math. 201, Birkhäuser (2001), 327-348.

[6] D. Joyce, Configurations in abelian categories. II. Ringel-Hall algebras, Adv. Math. 210(2), (2007), 635-706.

[7] A.D. King, Moduli of representations of finite-dimensional algebras, Quart. J. Math., Oxford Ser. (2) 45(180) (1994), 515-530.

[8] M. Kontsevich and Y. Soibelman, Stability structures, motivic Donaldson-Thomas invariants and cluster transformations, 2008.

[9] - Cohomological Hall algebra, exponential Hodge structures and motivic DonaldsonThomas invariants, Commun. Num. Theor. Phys. 5 (2011), 231-352.

[10] S. Mozgovoy, Poincaré polynomials of moduli spaces of stable bundles over curves, Manus. Math. 131(1-2) (2010), 63-86.

[11] - Motivic Donaldson-Thomas invariants and Kac conjecture, Comp. Math. 149 (2013), 495-504.

[12] — Wall-crossing formulas for framed objects, Quart. J. Math. 64 (2013), 489-513

[13] S. Mozgovoy and M. Reineke, On the noncommutative Donaldson-Thomas invariants arising from brane tilings, Adv. Math. 223(5) (2010), 1521-1544.

[14] K. Nagao, Donaldson-Thomas theory and cluster algebras, 2010.

[15] M. Reineke, The Harder-Narasimhan system in quantum groups and cohomology of quiver moduli, Invent. Math. 152(2) (2003), 349-368.

[16] — Counting rational points of quiver moduli, Int. Math. Res. Not. 17 (2006), ID 70456.

[17] — Localization in quiver moduli, J. Reine Angew. Math. 631 (2009), 59-83.

[18] E. Segal, The $A_{\infty}$ deformation theory of a point and the derived categories of local Calabi-Yaus, J. Algebr. 320(8) (2008), 3232-3268.

[19] T. Weist, Localization in quiver moduli spaces, Represent. Theory 17 (2013), 382-425.

School of Mathematics, Trinity College, Dublin 2, Ireland

E-mail address: mozgovoy@maths.tcd.ie 